\title{
Performance evaluation of sparse channel estimation for massive MIMO in LOS and NLOS environments
}

\author{
Jun Shikida ${ }^{\text {a) }}$ and Naoto Ishii \\ System Platform Research Laboratories, NEC Corporation, \\ 1753 Shimonumabe, Nakahara-ku, Kawasaki, Kanagawa 211-8666, Japan \\ a) j-shikida@cd.jp.nec.com
}

\begin{abstract}
Recently, a channel estimation method using channel sparsity in beam space has been studied. This method, which is called beam space channel estimation (BSCE), is effective for massive multiple-input multipleoutput (MIMO) systems because a larger number of antennas makes channel responses in beam space sparser. However, each of previous studies has used a specific channel model for evaluating the performance of BSCE although the channel model affects the performance. In this paper, we compare the performance of BSCE between line-of-sight (LOS) and non-LOS (NLOS) environments and clarify the effect of the difference in the channel sparsity on the performance.
\end{abstract}

Keywords: massive MIMO, multi-user MIMO, channel estimation Classification: Wireless Communication Technologies

\section{References}

[1] ARIB 2020 and Beyond Ad Hoc Group, "Mobile communications systems for 2020 and beyond," white paper, v 1.0.0, Oct. 2014.

[2] E. G. Larsson, O. Edfors, F. Tufvesson, and T. L. Marzetta, "Massive MIMO for next generation wireless systems," IEEE Commun. Mag., vol. 52, no. 2, pp. 186-195, Feb. 2014. DOI:10.1109/MCOM.2014.6736761

[3] S. L. H. Nguyen and A. Ghrayeb, "Compressive sensing-based channel estimation for massive multiuser MIMO systems," Proc. 2013 IEEE Wireless Communications and Networking Conference (WCNC), Shanghai, China, pp. 2890-2895, Apr. 2013. DOI:10.1109/WCNC.2013.6555020

[4] X. Xiong, X. Wang, X. Gao, and X. You, "Beam-domain channel estimation for FDD massive MIMO systems with optimal thresholds," IEEE Trans. Wireless Commun., vol. 16, no. 7, pp. 4669-4682, July 2017. DOI:10.1109/TWC.2017. 2701371

[5] J. Shikida, K. Muraoka, and N. Ishii, "Sparse channel estimation using multiple DFT matrices for massive MIMO systems," Proc. 2018 IEEE 88th Vehicular Technology Conference (VTC2018-Fall), Chicago, USA, Aug. 2018.

[6] 3GPP, TR 36.873 (V12.2.0), "Study on 3D channel model for LTE," July 2015. 


\section{Introduction}

To tackle the problem of increased mobile data traffic, research and development work for $5 \mathrm{G}$ mobile communications systems has been done [1]. To realize high speed communication required for the $5 \mathrm{G}$ systems, massive multiple-input multiple-output (MIMO) has been studied [2]. Massive MIMO is a transmission technology using a large number of antennas and can improve the performance of multi-user MIMO (MU-MIMO). For downlink (DL) MU-MIMO in a time division duplex (TDD) system, uplink (UL) channel estimation results are available for DL precoding because the channel reciprocity of the UL and DL holds. To avoid the performance degradation caused by channel estimation error, a channel estimation method using the sparsity of multipath channel in beam space has been studied $[3,4,5]$. This method, which is called beam space channel estimation (BSCE) in this paper, is effective for massive MIMO systems because a larger number of antennas makes channel responses in beam space sparser. To improve the estimation accuracy of BSCE, we have proposed a BSCE using multiple discrete Fourier transform (DFT) matrices for the space transformation of channel responses [5]. However, each of previous studies has used a specific channel model for evaluating the performance of BSCE although the channel model affects the performance.

In this paper, we evaluate the performance of BSCE in each of line-of-sight (LOS) and non-LOS (NLOS) environments and show the effect of the difference in the channel sparsity on the performance. We also evaluate the performance of the BSCE using multiple DFT matrices by increasing the number of DFT matrices than that used in [5].

\section{Beam space channel estimation}

The channel estimator using BSCE performs least squares (LS) channel estimation, antenna-to-beam space transformation, beam selection, and beam-to-antenna space transformation. Details on each of the above steps are described in the following subsections. Since the steps are performed independently for each user, user indexes are omitted in the following equations. In addition, the number of antennas of a user is assumed to be one to simplify the explanation.

\subsection{Least squares channel estimation}

First, the channel estimator estimates channel frequency responses for each antenna by using the LS schemes. Let $N$ and $h_{l}$ be the number of antennas of a base station (BS) and the channel frequency response between the $l$ th $(0 \leq l \leq N-1)$ antenna of the BS and the antenna of the user, respectively. The estimate of $h_{l}$ is given by

$$
\hat{h}_{l}=\frac{s_{\mathrm{RS}}^{*}}{\left|s_{\mathrm{RS}}\right|^{2}} \cdot y_{l}=h_{l}+\frac{s_{\mathrm{RS}}^{*}}{\left|s_{\mathrm{RS}}\right|^{2}} \cdot n_{l}
$$

where $s_{\mathrm{RS}}$ is the reference signal (RS) transmitted by the user, $y_{l}$ is the received signal in frequency domain, $n_{l}$ is the noise signal at the $l$ th antenna of the BS, and * represents the complex conjugate. 


\subsection{Antenna-to-beam space transformation}

Next, the channel estimator transforms the estimated channel responses from antenna to beam space. Let $N_{\mathrm{B}}$ be the number of beams. The $N_{\mathrm{B}}$-dimension vector whose elements correspond to the channel estimates in beam space is given by

$$
\hat{\mathbf{h}}_{\mathrm{B}}=\mathbf{A}^{\mathrm{H}} \hat{\mathbf{h}}
$$

where $\hat{\mathbf{h}}^{\mathrm{T}}=\left(\hat{h}_{0} \cdots \hat{h}_{N-1}\right), \hat{\mathbf{h}}_{\mathrm{B}}^{\mathrm{T}}=\left(\hat{h}_{\mathrm{B}, 0} \cdots \hat{h}_{\mathrm{B}, N_{\mathrm{B}}-1}\right), \mathbf{A}$ is the $N \times N_{\mathrm{B}}$ space transform matrix, ${ }^{\mathrm{H}}$ represents the Hermitian conjugate, and ${ }^{\mathrm{T}}$ represents the transpose.

Fig. 1(a) and (b) show the example of channel gain, which means the squared magnitude of channel response, in the antenna and beam space, respectively, where a DFT matrix is used as $\mathbf{A}$ and $N=N_{\mathrm{B}}=128$. As seen from Fig. 1(b), the channel responses in beam space at a BS side are generally regarded as approximately sparse because the angular spread at the BS side is often small. The channel sparsity in beam space holds well on the condition that the directions of beams are close to those of dominant paths. This condition is likely to be satisfied when the number of beams is large and the beams are formed in many directions.

To increase the probability that the directions of beams are close to those of dominant paths, we use multiple DFT matrices as the space transform matrix [5]. Let $Q, \mathbf{A}_{q}$, and $\mathbf{a}_{q, b^{\prime}}$ be the number of DFT matrices, the $q$ th $(0 \leq q \leq Q-1)$ DFT matrix, and the transform vector for the $b^{\prime}$ th $\left(0 \leq b^{\prime} \leq N-1\right)$ beam of $\mathbf{A}_{q}$, respectively. The space transform matrix $\mathbf{A}, \mathbf{A}_{q}$ and $\mathbf{a}_{q, b^{\prime}}$ are given by

$$
\begin{gathered}
\mathbf{A}=\left(\mathbf{A}_{0} \cdots \mathbf{A}_{Q-1}\right) \\
\mathbf{A}_{q}=\left(\mathbf{a}_{q, 0} \cdots \mathbf{a}_{q, N-1}\right) \\
\mathbf{a}_{q, b^{\prime}}^{\mathrm{T}}=\frac{1}{\sqrt{N}}\left[e^{j 2 \pi \cdot \frac{b^{\prime}+q / Q}{N} \cdot 0} \cdots e^{j 2 \pi \cdot \frac{b^{\prime}+q / Q}{N} \cdot(N-1)}\right]
\end{gathered}
$$

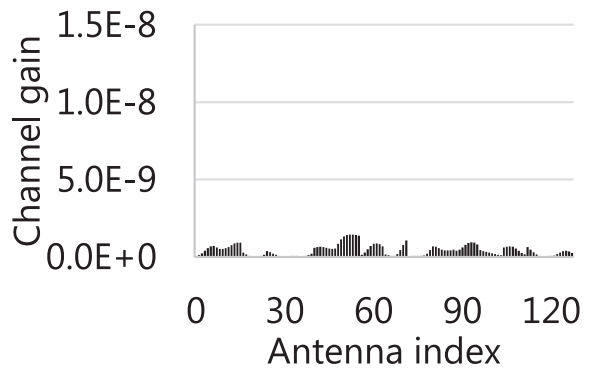

(a) Antenna space

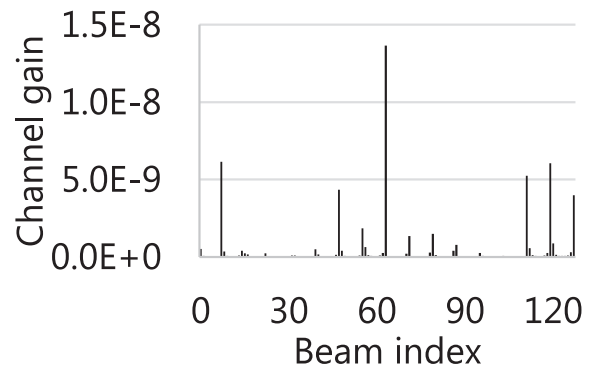

(b) Beam space

Fig. 1. Examples of channel gain $\left(N=N_{\mathrm{B}}=128\right)$

\subsection{Beam selection}

Then, the channel estimator selects non-zero beams from $N_{\mathrm{B}}$ beams and sets the channel estimates of unselected beams to zeros. Since channel responses in beam space are approximately sparse and noise power is almost equal among all beams, setting the channel estimates of non-dominant beams to zeros can reduce the estimation errors caused by the noise signals.

As the beam selection algorithm, we use threshold based algorithm because the computational complexity is relatively low $[4,5]$. The estimate of channel response 
between the $b$ th beam of the BS and the antenna of the user after beam selection is given by

$$
\tilde{h}_{\mathrm{B}, b}=\left\{\begin{array}{cc}
\hat{h}_{\mathrm{B}, b} & \text { if }\left|\hat{h}_{\mathrm{B}, b}\right|^{2}>\eta \sigma^{2} / P_{\mathrm{RS}} \\
0 & \text { otherwise }
\end{array}\right.
$$

where $\eta$ is a coefficient with a positive number, $\sigma^{2}$ is the noise power at the BS, and $P_{\mathrm{RS}}$ is the transmission power of the reference signal. Since $\sigma^{2} / P_{\mathrm{RS}}$ is equivalent to the variance of the channel estimation error (as seen from Eq. (1)), Eq. (6) means that the channel estimator selects beams whose squared magnitude of channel responses are higher than the $\eta$ times the estimation error variance. In the performance evaluation described below, $\eta$ is set to a value optimized for maximizing throughput.

\subsection{Beam-to-antenna space transformation}

Finally, the channel estimator transforms the channel estimates of the selected beams from beam to antenna space. The $N$-dimension vector calculated by the transformation is given by

$$
\tilde{\mathbf{h}}=\frac{1}{Q} \cdot \mathbf{A} \tilde{\mathbf{h}}_{\mathrm{B}}
$$

where $\tilde{\mathbf{h}}^{\mathrm{T}}=\left(\tilde{h}_{0} \cdots \tilde{h}_{N-1}\right)$ and $\tilde{\mathbf{h}}_{\mathrm{B}}^{\mathrm{T}}=\left(\tilde{h}_{\mathrm{B}, 0} \cdots \tilde{h}_{\mathrm{B}, N_{\mathrm{B}}-1}\right)$.

\section{Performance evaluation}

\subsection{Simulation conditions}

We evaluate the throughput performance when BSCE is used. The simulation conditions are as follows. The carrier frequency is $4.65 \mathrm{GHz}$. The number of BSs is one, and 30 users are uniformly distributed within 100-m radius and azimuth angle of $+/-60$ degrees from the BS. The BS has 128 antenna elements, which form a two-dimensional array [ 8 rows $\times 8$ columns $\times 2$ polarizations $(+/-45$ degrees $)$ ]. Each user has two antenna elements, which are cross-polarized antennas with 0 and 90 degree slant angles. The number of spatially multiplexed users is set to eight, and the number of layers per user is set to two. The combination of spatially multiplexed users is randomly selected. This is to prevent the user scheduling method from affecting the throughput performance. Zero forcing precoding is used because the computational complexity is relatively low compared with other precoding methods such as block diagonalization. The BS transmission power is set to $28 \mathrm{dBm}$ and is divided equally among spatially multiplexed layers. Although power allocation should be optimized for maximizing throughput, we use equal power allocation to simplify the analysis. The transmission period of UL RS is set to $10 \mathrm{~ms}$. 3GPP 3D urban micro (UMi) channel model is used [6]. In this model, mean angular spreads in azimuth at the BS side are 15.8 degrees for LOS and 25.7 degrees for NLOS. Mean angular spreads in zenith depend on the location of users and are about four to six degrees for both LOS and NLOS. 


\subsection{Throughput performance}

Figs. 2 and 3 show average cell throughput performance as a function of average signal-to-noise ratio (SNR) of UL-RS ( $\left.S N R_{\mathrm{UL}-\mathrm{RS}}\right)$ for LOS and NLOS environments, respectively. In these figures, the number of DFT matrices $Q$ for BSCE is set to one, four or nine. The performance of non-BSCE, which means the LS channel estimation only, is also plotted.

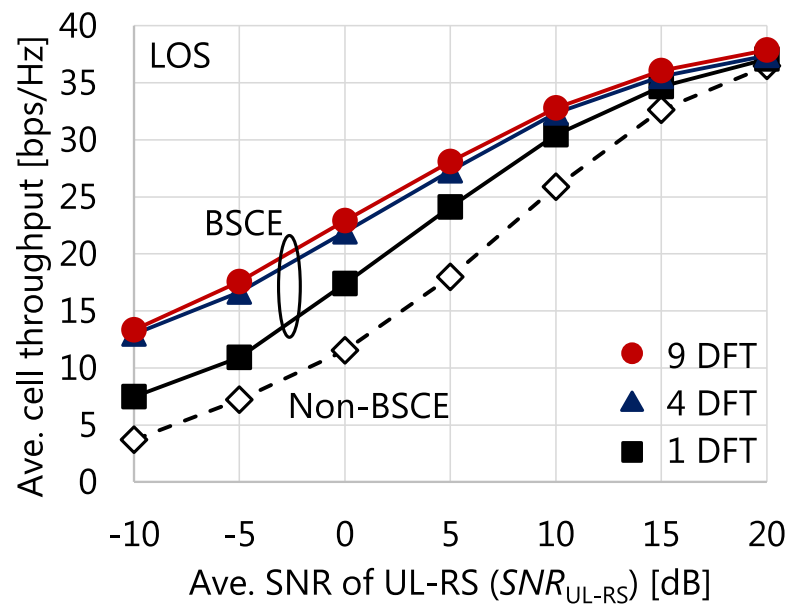

Fig. 2. Cell throughput performance for LOS

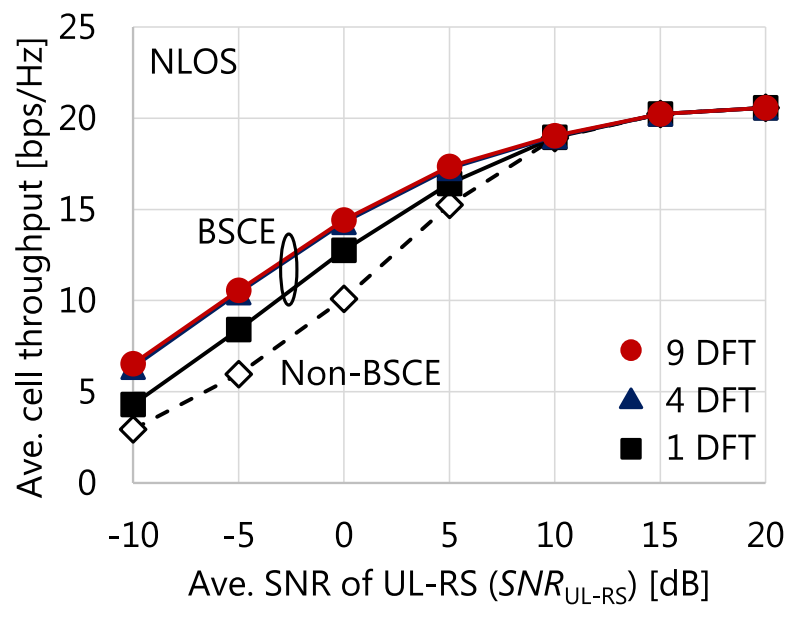

Fig. 3. Cell throughput performance for NLOS

For LOS environment, as seen from Fig. 2, cell throughput improvement compared with non-BSCE at $S N R_{\mathrm{UL}-\mathrm{RS}}=0 \mathrm{~dB}$ are $51 \%, 90 \%$, and $99 \%$ for one, four, and nine DFT matrices, respectively. It can be seen that the improvement when increasing the number of DFT matrices from four to nine is small regardless of $S N R_{\mathrm{UL}-\mathrm{RS}}$. This is because beams formed by four DFT matrices are sufficiently dense. It is also shown that the throughput improvement by BSCE decreases as $S N R_{\mathrm{UL}-\mathrm{RS}}$ increases and is a few percent at $S N R_{\mathrm{UL}-\mathrm{RS}}=20 \mathrm{~dB}$.

On the other hand, for NLOS environment, as seen from Fig. 3, cell throughput improvement compared with non-BSCE at $S N R_{\mathrm{UL}-\mathrm{RS}}=0 \mathrm{~dB}$ are $26 \%, 41 \%$, and $43 \%$ for one, four, and nine DFT matrices, respectively. It can be seen that the improvement for NLOS environment is smaller than that for LOS environment. 
This is because the channel response for NLOS is not sparser than that for LOS. It is also shown that BSCE has no throughput improvement compared with nonBSCE when $S N R_{\mathrm{UL}-\mathrm{RS}} \geq 10 \mathrm{~dB}$.

\section{Conclusion}

In this paper, we compared the performance of BSCE between LOS and NLOS environments and clarified the effect of the difference in the channel sparsity on the performance. Simulation results showed that cell throughput improvement by BSCE using one DFT matrix is $51 \%$ for LOS and $26 \%$ for NLOS when SNR of $\mathrm{UL}$ reference signal is $0 \mathrm{~dB}$. It was also shown that although using multiple DFT matrices for BSCE improved cell throughput, the improvement when increasing the number of DFT matrices from four to nine was small.

\section{Acknowledgments}

This paper includes a part of the results of "The research and development project for realization of the fifth-generation mobile communications system" commissioned by The Ministry of Internal Affairs and Communications, Japan. 